\title{
Challenging management of familial adenomatous polyposis with advanced colorectal cancer: laparoscopic partial resection and cold snare polypectomy
}

다(1)이우

\author{
Authors \\ Institutions \\ 1 Department of General Surgery, Tokyo-Nishi Tokushukai \\ Hospital, Akishima, Tokyo, Japan \\ 2 Department of Gastroenterology, Tokyo-Nishi \\ Tokushukai Hospital, Akishima, Tokyo, Japan
}

Shota Akabane ${ }^{1}$, Hiroaki Suda ${ }^{2}$, Hirokazu lijima ${ }^{1}$, Yukari Kobayashi ${ }^{1}$, Kazunao Watanabe ${ }^{1}$

submitted 15.3 .2018

accepted after revision 3.7.2018

Bibliography

DOI https://doi.org/10.1055/a-0677-1845 |

Endoscopy International Open 2019; 07: E49-E52

(c) Georg Thieme Verlag KG Stuttgart · New York ISSN 2364-3722

\section{Introduction}

Familial adenomatous polyposis (FAP) is caused by a germline mutation in the tumor suppressor gene, adenomatous polyposis coli, and accounts for approximately $1 \%$ of all colorectal cancers (CRCs) [1].

Most surgically untreated patients with FAP develop CRC in their lifetime. Prophylactic extended colectomy, subtotal colectomy with ileorectal anastomosis (IRA), or proctocolectomy with ileal pouch-anal anastomosis (IPAA) may be indicated. However, some patients refuse to undergo surgery because they are generally asymptomatic when diagnosed and removal of the large bowel affects their quality of life because of frequent diarrhea or loss of fecundity in women [2].

Endoscopic surveillance with polyp removal has been used to prevent development of CRC in patients with FAP. Recently, various polyp removal devices have appeared that have effectively shortened the duration of polypectomy, which has been beneficial for avoiding surgery [3]. However, the intervention for patients with FAP with advanced CRC is uncertain; therefore, extended colectomy is supposed to be performed [4].
Corresponding author

Shota Akabane, MD, Bessho 1-65-2, Hachioji, Tokyo, Japan

Fax: +81-42-674-5266

akap.sh.3381@gmail.com

\section{ABSTRACT}

Background and study aims Prophylactic extended colectomy may be indicated because most surgically untreated patients with familial adenomatous polyposis (FAP) develop colorectal cancer (CRC) in their lifetime. However, some patients refuse to undergo surgery to avoid degradation of their quality of life. We report that FAP is controllable with laparoscopic partial resection and postoperative polypectomy even when complicated by advanced CRC. We also discuss the utility of cold snare polypectomy for resection of polyps.

Here, we report that FAP is controllable with laparoscopic partial resection and postoperative polypectomy even when it is complicated by advanced CRC.

\section{Case report}

A 39-year-old Japanese woman presented with abdominal pain and diarrhea that had persisted for a month. She also experienced vomiting and hematochezia on the day of the visit. Her medical history was unremarkable. However, her father and older sister had been diagnosed with FAP and had undergone proctocolectomy with IPAA for adenocarcinoma of the sigmoid colon with polyposis. On physical examination, there was tenderness in the lower abdomen and the woman's body temperature was $36.5^{\circ} \mathrm{C}$. Laboratory tests were negative for anemia and tumor markers. On the other hand, abnormal glucose tolerance was revealed (blood sugar level $330 \mathrm{mg} / \mathrm{dL}$, HbA1c 7.2\%). Enhanced computed tomography (CT) scan showed thickness of the sigmoid colon wall, but no lung or liver metastases were detected. Colonoscopy showed a nearly obstructing tumor in the sigmoid colon and polyposis ( $\triangleright$ Fig.1a, $>$ Fig. 1b, $>$ Fig.1c). 

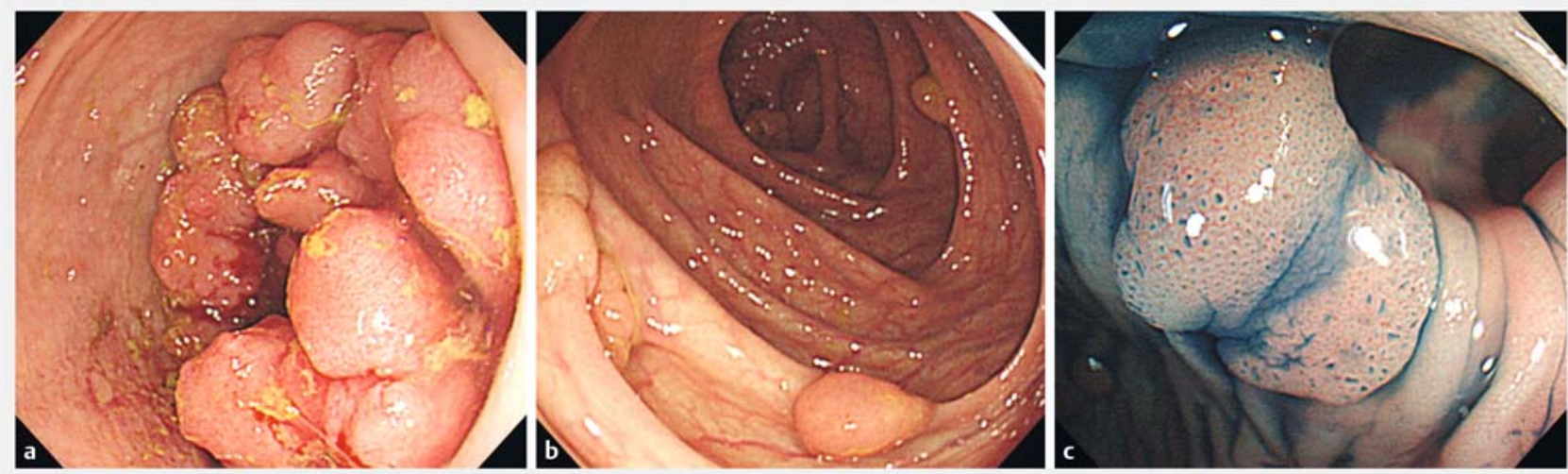

- Fig. 1a Colonoscopy showed a nearly obstructing tumor in the sigmoid colon and $\mathbf{b}$ polyposis. $\mathbf{c}$ Indigo carmine stain highlighted a typical pit pattern characterized as adenomas.

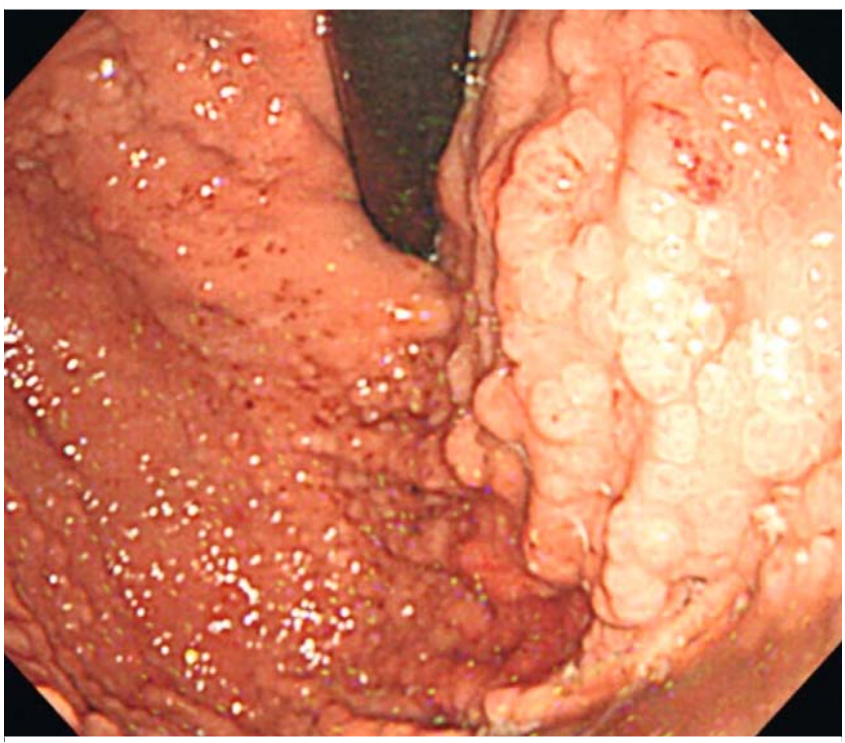

- Fig. 2 Esophagogastroduodenoscopy showed many fundic gland polyps from the fornix to the upper body of the stomach.

Using a miniature endoscope to pass the tumor and observe to the cecum, we detected approximately 100 polyps (20 in the rectum). Cytology of the tumor showed well-differentiated adenocarcinoma. Esophagogastroduodenoscopy showed many fundic gland polyps from the fornix to the upper body of the stomach ( $\mathbf{F i g . 2}$ ). Based on the patient's clinical course and family history, she was subsequently diagnosed with advanced sigmoid colon adenocarcinoma because of attenuated FAP (aFAP). We also performed thyroid ultrasound and positron emission CT to exclude extracolonic manifestations such as desmoid tumors. At first, proctocolectomy with IPAA was considered to cure both the tumor and the polyposis, but the patient refused surgery because she wished to keep working full time and bear children. Therefore, we decided to perform laparoscopic partial sigmoidectomy for treatment of the adenocarcinoma, followed by endoscopic polypectomy to control the burden of polyposis. Laparoscopic surgery was performed with five ports, and D3 lymph node resection was accomplished as well. Transanal anastomosis was performed with an EEA stapler with DST Series Technology $25 \mathrm{~mm}$ (Medtronic, Minneapolis, Minnesota, United States). Postoperative polypectomy was mainly performed by cold snare polypectomy (CSP) with PCF-H 290 (Olympus, Japan) and CAPTIVATOR II Single-Use Snare (Boston Scientific, Marlborough, Massachusetts, United States) ( $\triangleright$ Fig.3a and Fig.3b). We resected approximately 100 polyps during five procedures (average duration of procedure, 31 min; range, $22-45 \mathrm{~min}$ ) without any complications such as postoperative bleeding or perforation. The number of polyps has been decreasing under our periodic endoscopic procedures and almost all the polyps were diagnosed with adenoma pathologically. In addition, eight courses of capecitabine (oral; $1250 \mathrm{mg} / \mathrm{m}^{2}$ twice daily for 2 weeks) were administrated based on the pathological findings of the adenocarcinoma: PT3N1M0 stage IIIB according to the 7th Union for International Cancer Control classification. We also administered metformin hydrochloride (oral; $500 \mathrm{mg}$ twice daily) to control not only blood sugar level but also polyp burdens.

The patient has been followed up more than 5 years without any recurrence of CRC or increase in number of polyps. She has been working as before without any complaints.

\section{Discussion}

This case highlights two important issues. First, even when it is complicated by CRC, FAP is controllable by laparoscopic partial resection and postoperative polypectomy, thus delaying the need for extended colectomy. Second, CSP is a very useful and sufficient procedure for resecting FAP polyps.

It is well known that colectomy is indicated for patients with FAP who have CRC or adenoma with high-grade dysplasia. Generally, extended colectomy, subtotal colectomy with IRA, or proctocolectomy with IPAA is performed, depending on the severity and distribution of colorectal adenomas. In patients with fewer than 10 rectal adenomas, subtotal colectomy with IRA is preferred because rectal polyps can be managed endoscopically [5]. Other important factors to consider include risk of des- 


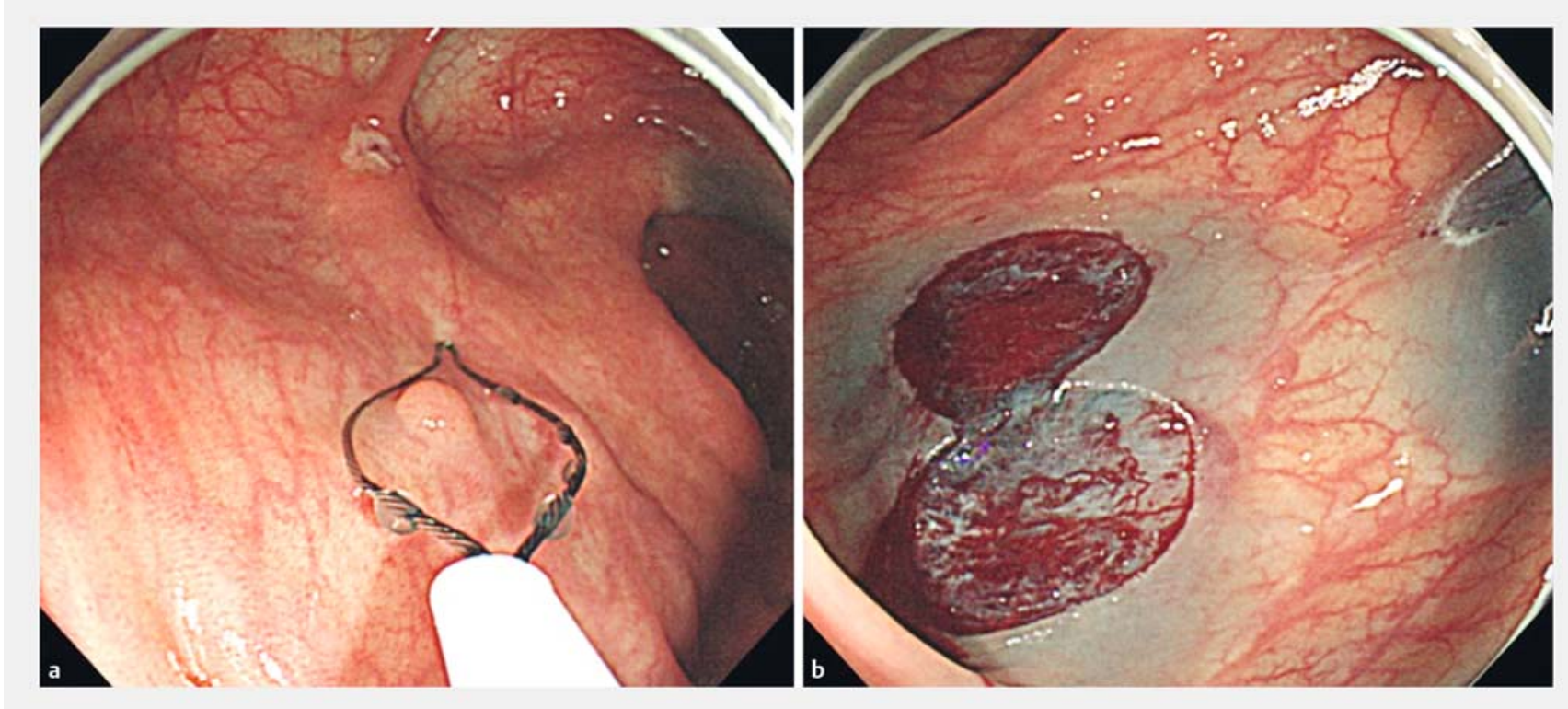

Fig. 3 a Postoperative polypectomy was mainly performed by cold snare polypectomy (CSP). b It can be performed with submucosal infusion, if necessary.

moid tumors and patient age and comorbidities [6]. On the other hand, it has been suggested that endoscopic polypectomy can be considered in management of aFAP, which is characterized by fewer adenomas, later age of onset for colorectal adenomas and cancer, and decreased risk of cancer compared with typical FAP [7]. In this case, although the patient was diagnosed with aFAP with advanced sigmoid adenocarcinoma and proctocolectomy with IPAA was recommended because of her clinical course, family history, and poly distribution, she strongly refused this treatment.

To the best of our knowledge, this is the first report of management of FAP with advanced CRC with laparoscopic partial resection and postoperative polypectomy.

To resect numerous polyps effectively, two cold polypectomy techniques are available. Cold forceps polypectomy (CFP) is safe and easy to perform without the need for special techniques or an experienced assistant. A second option, CSP, has also been reported to be a safe and effective for endoscopic resection of small colorectal polyps [3].

CSP has been established as an effective method for removal of diminutive and small polyps [3]. An advantage of CSP over CFP is that capturing from one to a few millimeters of normal mucosa surrounding the polyp is more likely to result in complete removal. The technique also causes minimal damage to the submucosal arteries and can be performed safely in patients taking antiplatelet agents or therapeutic levels of anticoagulants [8]. Although there were concerns that it would be difficult to retrieve tissues with the CSP technique, it is now known that tissue retrieval after the CSP technique is successful more than $94 \%$ of the time [9]. In addition, a prospective study indicated that CSP is superior to CFP for complete histologic eradication of polyps (93.2\% vs. $75.9 \%, P=0.009)$ with excellent safety and acceptable tissue retrieval [10].
A retrospective review identified a large group of patients with FAP who strongly refused to undergo colectomy [4]. These patients were managed endoscopically and underwent repeated colonoscopies to remove numerous polyps with the aim of colon clearance. During a median follow-up of 5.1 years, no invasive CRC was observed. Five patients had noninvasive carcinomas that were detected within 10 months from the start of the follow-up period. All of these patients were treated endoscopically, and they showed no signs of recurrence during follow-up.

In the case described here, we diagnosed the patient with aFAP based on her clinical course including family history. We didn't check the type of genetic mutations because health insurance in japan does not provide compensation for that testing. Approximately 100 polyps were resected by CSP, and almost all of them were completely removed with normal mucosa surrounding the polyps in a relatively short time without any complications. CSP can satisfy requirements for histological eradication, safety, and promptness when dealing with huge numbers of polyps. It is essential to perform periodic colonoscopy and polypectomy in addition to whole-body follow-up because the follow-up period may be insufficient oncologically, and the long-term outcome is unknown. In this case, the patient's postoperative clinical course such as the number of polyps, subjective symptoms, and findings in other modalities has improved during observation.

Although management of aFAP with advanced cancer by laparoscopic partial resection and postoperative CSP is challenging, it can be an option for patients who refuse extended colectomy. 


\section{Conclusion}

Even when it is complicated by CRC, FAP is controllable by laparoscopic partial resection and postoperative polypectomy, thus delaying need for extended colectomy. To deal with large numbers of polyps, CSP is a very useful and sufficient procedure, satisfying requirements for histological eradication, safety, and promptness.

\section{Acknowledgements}

The authors thank Crimson Interactive Pvt. Ltd. for revision and review of the manuscript.

Competing interests

None

\section{References}

[1] Groden J, Thliveris A, Samowitz W et al. Identification and characterization of the familial adenomatous polyposis coli gene. Cell 1991; 66: $589-600$
[2] Olsen $\mathrm{K} \varnothing$, Juul S, Bülow $S$ et al. Female fecundity before and after operation for familial adenomatous polyposis. Br J Surg 2003; 90: 227 231

[3] Patel NJ, Ponugoti PL, Rex DK et al. Cold snare polypectomy effectively reduces polyp burden in familial adenomatous polyposis. Endosc Int Open 2016; 4: E472 -E474

[4] Ishikawa H, Mutoh M, Iwama T et al. Endoscopic management of familial adenomatous polyposis in patients refusing colectomy. Endoscopy 2016; 48: $51-55$

[5] Vasen HF, Möslein G, Alonso A et al. Guidelines for the clinical management of familial adenomatous polyposis (FAP). Gut 2008; 57: 704

[6] Nieuwenhuis MH, Mathus-Vliegen LM, Slors FJ et al. Genotype-phenotype correlations as a guide in the management of familial adenomatous polyposis. Clin Gastroenterol Hepatol 2007; 5: 374- 378

[7] Burt RW, Leppert MF, Slattery ML et al. Genetic testing and phenotype in a large kindred with attenuated familial adenomatous polyposis. Gastroenterology 2004; 127: 444-451

[8] Horiuchi A, Nakayama Y, Kajiyama M et al. Removal of small colorectal polyps in anticoagulated patients: a prospective randomized comparison of cold snare and conventional polypectomy. Gastrointest Endosc 2014; 79: 417-423

[9] Deenadayalu VP, Rex DK. Colon polyp retrieval after cold snaring. Gastrointest Endosc 2005; 62: 253-256

[10] Lee CK, Shim JJ, Jang JU et al. Cold snare polypectomy vs. Cold forceps polypectomy using double-biopsy technique for removal of diminutive colorectal polyps: a prospective randomized study. Am J Gastroenterol 2013; 108: 1593 